Meta

Journal des traducteurs

Translators' Journal

\title{
Table ronde sur l'enseignement de la traduction
}

\section{Jean Delisle, Raymond Frenette, Gilles Gervais, Paul A. Horguelin, Aline Manson-Daoust et Francis Whyte}

Volume 20, numéro 1, mars 1975

Vingt ans de traduction. Bilan et prospectives

URI : https://id.erudit.org/iderudit/002511ar

DOI : https://doi.org/10.7202/002511ar

Aller au sommaire du numéro

\section{Éditeur(s)}

Les Presses de l'Université de Montréal

ISSN

0026-0452 (imprimé)

1492-1421 (numérique)

Découvrir la revue

Citer cet article

Delisle, J., Frenette, R., Gervais, G., Horguelin, P. A., Manson-Daoust, A. \& Whyte, F. (1975). Table ronde sur l'enseignement de la traduction. Meta, 20(1), 42-57. https://doi.org/10.7202/002511ar 


\section{Table ronde sur l'enseignement de la traduction}

Sil est possible de faire en trois heures et demie un tour d'horizon de l'enseignement de la traduction au Canada, résumer en quelques pages la riche moisson d'informations et d'opinions ainsi glanée est un défi que je n'ai pas la prétention d'avoir relevé. J'espère donc que les participants voudront bien m'excuser d'avoir condensé à l'extrême leurs interventions pour n'en retenir que la quintessence.

La formation des traducteurs est une préoccupation partagée par les écoles de traduction, leurs professeurs et étudiants, mais aussi par les associations professionnelles et les employeurs des secteurs public et privé. Je me suis donc efforcé d'associer ces différents milieux à nos échanges de vue, sans oublier nos aimables consœurs. La table ronde était ainsi constituée des personnes suivantes, dont les initiales permettront l'identification :

J. D. M. Jean Delisle, professeur à l'École de traducteurs et d'interprètes de l'Université d'Ottawa.

R. F. M. Raymond Frenette, directeur des services linguistiques de la Sun Life ; vice-président de la Société des traducteurs du Québec.

G. G. M. Gilles Gervais, étudiant à l'École de traduction de l'Université de Montréal.

P. H. M. Paul A. Horguelin, professeur à l'École de traduction de l'Université de Montréal (animateur).

A. M. D. $M^{\text {me }}$ Aline Manson-Daoust, réviseur au Bureau fédéral des traductions à Montréal.

F. W. M. Francis Whyte, directeur du module Langues modernes, Université du Québec à Trois-Rivières.

Deux représentants de l'Antenne, organe de la S.T.Q., ont assisté à notre rencontre à titre d'observateurs ; ce sont MM. Gilles Breton et Pierre Marchant.

À tous, je désire exprimer mes vifs remerciements pour leur cordiale et fructueuse participation. Je remercie aussi les directeurs et responsables d'écoles de traduction qui ont bien voulu me fournir un bref historique de l'enseignement dans leur université.

Paul A. Horguelin 
P. H. - Avant de procéder à un tour d'horizon de l'enseignement de la traduction au Canada, il serait peut-être utile de tracer un bref historique de cet enseignement.

Les premiers cours, donnés le soir, ont été créés en 1936 à l'Université d'Ottawa. Essentiellement pratiques, ils s'adressaient à des traducteurs fédéraux et aux personnes qui se préparaient à subir l'examen de la Commission de la fonction publique. Vers 1940 , on assiste à la création presque simultanée de cours du soir à l'Université McGill et à l'Institut de traduction de Montréal. Ces cours étaient suivis par des adultes, pour la plupart traducteurs ou secrétaires-traductrices; ils étaient sanctionnés par un diplôme et non par un grade universitaire ; enfin, on enseignait la traduction dans les deux sens (anglais-français et vice-versa). Cet enseignement se poursuit de nos jours au Centre d'éducation permanente de l'Université McGill et au Service d'éducation permanente de l'Université de Montréal, qui a intégré l'Institut de traduction en 1965. L'Université Laval offre aussi un cours du soir réparti sur quatre ans. L'Université de Sherbrooke, qui avait créé un certificat de traduction en 1972 (30 étudiants), abandonnera cet enseignement à la fin de 1975 .

Les premiers cours du jour remontent à 1950, année de la création d'une Section de traduction à l'Université de Montréal. Le programme comportait à l'origine deux années d'étude et la rédaction d'une thèse ; le diplôme décerné était une maîtrise en traduction. En 1968, le programme a été porté à trois ans; le diplôme est devenu une licence, puis un baccalauréat spécialisé. Enfin, une nouvelle maîtrise est venue compléter l'enseignement dispensé en permettant un début de spécialisation. Le nombre des étudiants est passé d'une dizaine en 1950 à 320 en 1974. Devenue École de traduction, la section compte 37 professeurs, dont 22 à la leçon.

Toujours au Québec, l'Université Laval offre un programme de trois ans conduisant à un baccalauréat spécialisé en traduction. Une centaine d'étudiants s'inscrivent, chaque année, et on espère créer prochainement une maîtrise en traduction. L'Université du Québec à Trois-Rivières a un programme de baccalauréat en traduction et projette la création d'une maîtrise en terminologie; elle compte 4 professeurs à temps complet et 70 étudiants.

En Ontario, l'enseignement de la traduction à l'Université d'Ottawa a d'abord été donné dans le cadre d'une maîtrise en linguistique ; il est assuré depuis 1971 par l'École de traducteurs et d'interprètes qui offre actuellement un programme de premier cycle et compte réintroduire un programme de maîtrise en 1976. L'École compte 6 professeurs à temps complet, 18 professeurs à la leçon et 200 étudiants. De son côté, l'École des traducteurs et interprètes de l'Université Laurentienne à Sudbury dispense un enseignement bilingue de la traduction et de l'interprétation réparti sur quatre ans et sanctionné par un baccalauréat scientifique en langues.

Au Nouveau-Brunswick, l'Université de Moncton a créé en 1972 un baccalauréat en traduction pour remédier à la pénurie de traducteurs professionnels dans cette province. Le programme comprend une année de préparation et trois années de spécialisation. On envisage de former une quinzaine de diplômés par an. 
À ces universités qui dispensent un enseignement complet, il faudrait ajouter celles qui offrent des cours de traduction dans le cadre d'autres programmes : Sir George Williams, Toronto et Manitoba. Ces faits et chiffres nous aideront à répondre à une première question :

\section{Peut-on enseigner la traduction?}

Cette question, que le professeur J.-P. Vinay pouvait encore poser en 1957 dans le Journal des traducteurs, paraît quelque peu dépassée lorsqu'on considère la multiplication des écoles de traduction depuis vingt ans. Posons-la néanmoins, ne serait-ce que pour rappeler que les directeurs des premières écoles ont eu à y répondre souvent et que, de nos jours encore, il se trouve des personnes - notamment parmi les self-made traducteurs - pour qui la réponse n'est pas évidente. N'oublions pas non plus que certains étudiants, à leur retour de stage, déclarent que la formation «sur le tas» est plus profitable que les études, constatation que semblent confirmer les statistiques d'engagement au Bureau fédéral des traductions : le taux de réussite des diplômés et des non-diplômés est sensiblement le même. La vraie question à poser est peut-être alors : Faut-il enseigner la traduction?

F. W: - C'est une question fondamentale et qui s'est souvent discutée. Il est évident qu'il y a d'excellents traducteurs parmi les autodidactes, mais ce que nous essayons de faire dans les universités, c'est de donner à des étudiants qui n'ont pas ce bagage-là le jugement et l'esprit analytique leur permettant d'acquérir plus vite, «sur le terrain », la compétence nécessaire. Quant au taux de réussite, il ne faut pas oublier que les diplômés sont en général plus jeunes que les non-diplômés engagés par le Bureau des traductions, et on peut espérer qu'à âge égal ils feront de meilleurs traducteurs.

J. D. - On a posé la même question dans le cas d'autres disciplines. Je songe, par exemple, au journalisme. Certaines personnes estiment que ce métier s'apprend uniquement dans une salle de rédaction, d'autres, au contraire, sont convaincues qu'il peut faire l'objet d'un enseignement universitaire. À mon avis, il n'y a pas qu'un seul chemin qui mène à la traduction. Une minorité de candidats ont une grande facilité à faire passer un message d'une langue dans une autre. Les cours de traduction s'adressant plus spécialement à tous les autres qui ne sont pas doués du même talent naturel. La traduction a donc sa place parmi les disciplines universitaires.

P. H. - Il y a aussi un facteur historique. Jusqu'au $\mathrm{xx}^{\mathfrak{e}}$ siècle, qui disait 《traduction» pensait «traduction littéraire », d'où la conception de la traduction comme un art. Depuis Fëdorov, Mounin, Vinay et Darbelnet, la traduction revendique le titre de science ou, soyons modestes, de discipline. Et ses techniques peuvent s'enseigner.

J. D. - En effet, il ne faut pas oublier qu'au Canada, la traduction est beaucoup plus utilitaire. Et chaque genre de communication a une forme, un style particulier. Ces choses-là s'enseignent. 
A. M. D. - Je confirmerais ce que vous dites, en me plaçant du point de vue du réviseur. Dans le cas des diplômés, il n'est généralement pas nécessaire de leur apprendre tous les principes de base. Pour les autres, par contre, il faut leur donner la formation qu'ils n'ont pas reçue à l'école, notamment en ce qui concerne la langue et les techniques de la traduction. Eux-mêmes confirment d'ailleurs cette observation en s'inscrivant à des cours du soir en traduction.

P. H. - Monsieur Gervais, à titre d'étudiant qui a déjà fait deux stages, pouvez-vous établir une comparaison entre les deux modes de formation? Se complètent-ils ou l'un peut-il remplacer l'autre ?

G. G. - L'un ne remplace pas l'autre, mais il est vrai que, comme beaucoup de boursiers du gouvernement fédéral, j'ai appris deux fois plus en six mois de stage qu'en deux années d'étude. Il n'y a pas incompatibilité entre théorie et pratique, mais l'enseignement de l'un est trop déphasé par rapport à la réalité de l'autre.

P. H. - Autrement dit, vous n'arrivez pas à établir un rapport entre l'enseignement, disons théorique, qu'on vous donne, bien qu'il nous paraisse pratique, et son application au milieu du travail ?

G. G. - Non. La différence entre la théorie et la pratique est trop grande.

J. D. - Le but de l'enseignement de la traduction n'est pas de former des « virtuoses » pour qui la traduction n'a plus de secrets. Les Écoles de traduction se sont fixé des objectifs beaucoup plus modestes : d'une part, dispenser un enseignement correctif portant sur les impropriétés susceptibles d'entacher un texte traduit ; d'autre part, aiguiser le sens critique et analytique, le jugement et le bon goût des étudiants tout en forçant ces derniers à s'astreindre à une discipline rigoureuse de travail. Les Écoles cherchent aussi à élargir le plus possible les connaissances des étudiants et à développer chez eux un sens aigu de l'observation et une grande souplesse de style. Je conçois l'enseignement de la traduction comme une «mise en train » qui prépare de futurs artisans de la langue à assumer le mieux possible leurs fonctions d'intermédiaires en communication écrite. Mais il restera toujours des lacunes à combler.

G. G. - J'aimerais que $\mathrm{M}^{\text {me }}$ Manson-Daoust nous dise combien il faut de temps à un traducteur non diplômé pour combler ces lacunes.

A. M. D. - À peu près un an, tout comme il faut aussi environ un an pour que le diplômé s'adapte au milieu du travail. Je dois ajouter qu'il y a autant de bons que de mauvais éléments dans les deux groupes et préciser que les traducteurs non diplômés engagés par le gouvernement sont pour la plupart d'anciens professeurs et journalistes.

R. F. - Quelqu'un à dit : « Rien ne' s'enseigne, tout s'apprend. » C'est peutêtre paradoxal, mais vrai, du moins pour la formation pratique. Et le même problème se pose pour tous les professionnels qui quittent l'université et arrivent en situation réelle. Le jeune traducteur ne doit donc pas s'illusionner, ni se sentir frustré ; mais il faudrait le lui dire au départ. Je pense que la première année est un peu affolante. Le traducteur a l'impression de ne rien savoir, il manque de 
confiance, au point que M. Deschamps, de Bell Canada, me disait récemment que les nouveaux diplômés en viennent à vérifier l'orthographe de mots courants... Ce n'est qu'au bout d'un certain temps que le traducteur se rend compte à quel point ses études ont été utiles. Mais, au début, il se perd dans le quotidien, dans le feu de l'action.

F. W. - Je pense que cet «affolement » du traducteur n'a rien d'étonnant; comme on l'a dit, le jeune médecin et le jeune avocat vivent la même expérience. Mais, ce qui distingue la traduction - et est au coeur du problème que soulève la formation - c'est que, contrairement à la médecine ou au droit, il y a des personnes qui deviennent traducteurs sans passer par l'université. Il faut donc se poser la question : qu'est-ce qui distingue un traducteur professionnel de quelqu'un qui fait de la traduction? Je pense qu'à l'université nous tentons de former des traducteurs professionnels, c'est-à-dire des personnes qui soient capables de s'adapter à des contextes très divers, de se recycler, de faire rapidement un travail de qualité. Si on prétend que la traduction est une profession, et c'est le but visé par les associations professionnelles, il faudra à un moment donné préciser ce qui différencie le professionnel de l'amateur. C'est loin d'être clair.

P. H. - En résumé, on peut donc dire qu'il est possible d'enseigner la traduction, mais que l'enseignement ne peut remplacer l'apprentisage dans le milieu de travail, les deux modes de formation se complétant. Ce point étant acquis, une autre question plus complexe vient logiquement à l'esprit :

\section{Comment enseigner la traduction?}

J. D. - On se heurte à un problème considérable, du fait que la traduction est une discipline qui embrasse tout le champ des connaissances... Il faut donc se restreindre. Apprendre à traduire, c'est essentiellement apprendre à maîtriser la langue d'arrivée dans ses applications pratiques aux différents domaines. Un contrat d'assurance ne se traduit pas comme une annonce publicitaire. Le bilingue qui traduit se contente souvent de transmettre l'idée ; le traducteur doit être plus précis et transmettre le message intégral en respectant la forme de la langue d'arrivée. C'est ce qui distingue le traducteur professionnel du bilingue et du charlatan.

F. W. - Il faut que le traducteur soit conscient du milieu, du contexte professionnel, et les programmes d'enseignement devraient refléter cet aspect. Comment enseigner la traduction? Je pense que personne ne le sait exactement. On procède de façon ad hoc, en fonction de certaines contraintes, de l'évolution des besoins, mais rien n'est systématique. L'enseignement actuel de la traduction au Canada est façonné par le contexte canadien; en ce sens, il est très spécifique.

J. D. - À cela s'ajoute l'omniprésence de la traduction au Canada où on est littéralement submergé de textes traduits. $\mathrm{La}$ traduction y est devenue un véritable mode de penser. La qualité de la langue au pays dépend dans une large mesure de la qualité de la traduction, donc de la qualité des traducteurs professionnels. L'enseignement de la traduction doit donc être surtout pratique et toucher au plus grand nombre de domaines possible. 
A. M. D. - Je me permettrai quelques critiques. Il me semble que les professeurs n'ont pas assez de recul par rapport à la traduction qu'ils enseignent; ils n'ont pas vraiment réfléchi sur le processus de la traduction et ne peuvent donc l'expliquer aux étudiants. L'étudiant ne comprend pas ; il ne retient que les recettes de cuisine. De son côté, le réviseur n'a souvent pas la formation nécessaire pour enseigner autre chose que des recettes. En définitive, tout le monde enseigne des recettes ; il n'y a jamais un véritable enseignement théorique.

F. W. - Je suis absolument d'accord. Je pense aussi qu'en plus de la connaissance des langues, l'enseignement devrait porter sur la formation du jugement, de l'esprit de traducteur.

P. H. - Vous venez de faire ressortir l'importance d'un véritable enseignement théorique de la traduction. Or, les étudiants et certains employeurs nous reprochent - à tort ou à raison — d'être trop théoriques...

G. G. - J'ai vraiment l'impression qu'on nous enseigne des recettes, simplement parce que chaque professeur, et je ne mets pas en doute leur compétence professionnelle, semble détenir la vérité. L'enseignement est boîteux et nous prépare mal. Je me demande si les professeurs savent ce qu'est l'enseignement de la traduction. La pédagogie est un sujet tabou dans ce milieu.

P.H. - Est-ce que vous considérez que la linguistique différentielle, la stylistique comparée de Vinay-Darbelnet sont des recettes?

G. G. - C'est souvent ainsi qu'on nous présente ces matières.

A. M. D. - En fait, il n'y a que quelques professeurs qui les enseignent. On pourrait croire que certains étudiants n'en ont jamais entendu parler.

F. W. - Je pense que les recettes sont nécessaires, mais elles devraient s'apprendre en stage. Dans les cours de traduction, on devrait préparer l'étudiant à recevoir la formation pratique dans le milieu du travail. On s'illusionne si on pense qu'on peut donner cette formation pratique à l'université : le temps passé en classe est minime comparativement à ce que fait le traducteur professionnel.

J. D. - Vous proposez donc des exercices pratiques de réflexion sur les difficultés et les mécanismes de la traduction?

G. G. - Il faut que les étudiants apprennent les mécanismes, c'est une nécessité, mais si cela consiste à s'extasier devant tel ou tel processus de traduction, ils ne marchent plus, et pour cause.

P. H. - Nous avons tenté jusqu'ici de concilier formation théorique et formation pratique. Un autre aspect à considérer est celui de la formation générale opposée à la formation spécialisée. Les étudiants expriment souvent le désir d'être «rentables» dès leur sortie de l'université et font valoir que sur le marché du travail on demande surtout des traducteurs spécialisés.

R.F. - Ce qu'on demande à l'université, c'est de compléter la formation générale que les étudiants sont censés avoir acquise au niveau collégial, de leur enseigner les principes de base de la traduction, d'encourager chez eux l'esprit de 
curiosité, de les inciter à lire et à se tenir au courant de ce qui se passe autour d'eux. On devrait aussi leur apprendre une méthodologie : comment aborder un travail de traduction. Peut-être leur donner moins de textes à traduire, mais leur faire prendre conscience de certaines contraintes du milieu de travail, notamment les délais et le rendement. Tout cela est loin de la spécialisation. Je pense qu'il est prématuré et dangereux de spécialiser les étudiants à l'université ; les employeurs sont souvent mieux placés pour le faire. En outre, on ne sait pas quelle sera la demande dans dix ans, dans cinq ans même.

J. D. - En effet, l'étudiant en traduction n'a pas les données nécessaires pour choisir sa spécialisation.

A. M. D. - Je ne suis pas tout à fait d'accord. La spécialisation en cours d'emploi est peut-être idéale, mais dans la majorité des cas elle n'est pas réaliste. D'une part, le jeune traducteur n'a pas le temps d'acquérir cette formation de façon systématique pendant les heures de travail, et après avoir traduit pendant sept heures et demie, il n'a guère envie de se remettre à l'étude. D'autre part, beaucoup d'employeurs n'ont pas de personnel qualifié pour assurer cette formation. Je pense donc que les écoles de traduction devraient initier les étudiants à certains domaines spécialisés, sans chercher à en faire des spécialistes, ce qui est impossible. Plutôt que de leur faire traduire des textes souvent vagues, leur enseigner une méthode de travail, leur indiquer où trouver la documentation. Bref, les placer en situation réelle : «Vous avez ce texte juridique à traduire, qu'est-ce que vous faites? ?Etre spécialisé, cela veut aussi dire savoir de quol l'on parle, et je pense qu'on devrait offrir la possibilité de suivre des cours d'économie, de droit, aux étudiants pendant leur scolarité de traduction. Cependant, il faut veiller à ce que ces cours soient adaptés aux besoins du traducteur. Il est inutile, par exemple, d'enseigner les régimes matrimoniaux; par contre, l'enseignement des principes de Common Law et de droit civil est de première importance. Autre exemple : enseigner les fondements de l'économie de marché, mais par les différents modèles d'économie qui existent.

F. W. - Personne, je crois, ne prétend former des traducteurs juridiques en 45 heures. L'important, c'est que l'étudiant apprenne les techniques qui lui permettent d'aborder un domaine spécialisé où, à première vue, il comprend peu de chose.

J. D. - Si on prend l'exemple de l'informatique, il est inutile que le traducteur sache programmer un ordinateur, mais il doit avoir certaines notions de programmation. On pourrait ajouter aux programmes de traduction une liste de lecture d'ouvrages de base dans des domaines de spécialisation. L'étudiant pourrait ainsi étendre ses connaissances tout en acquérant la terminologie propre aux domaines étudiés.

P. H. - Vous conviendrez sans doute que le meilleur programme de traduction reste une vue de l'esprit s'il ne tient pas compte de ce que j'appellerais, sans vouloir offenser personne, la «matière première» et le «produit fini ». Pouvonsnous essayer de tracer le portrait-robot de l'étudiant qui s'inscrit en traduction? 


\section{La «matière première »}

G. G. - Je crois que beaucoup d'étudiants qui sortent des cégeps s'inscrivent en traduction parce qu'ils ne savent pas où aller. Ils ont une idée très vague de ce qu'est, en pratique, le métier de traducteur, et très souvent cette idée n'est pas plus précise à la fin de leurs études.

P. H. - C'est là un premier aspect important: le manque d'information préalable. Le deuxième aspect est le bagage acquis avant l'entrée à l'université.

G. G. - Les étudiants ont suivi tous les cours de français : poésie, théâtre, roman canadien... et tous les cours d'anglais. Mais le problème reste le même, étant donné qu'ils ne savent pas, et les cégeps non plus, ce qu'on attend d'eux à l'université. Alors, on tente l'expérience.

P. H. - À la lumière de cette expérience, qu'est-ce qui vous a manqué ?

G. G. - L'information. Il n'existe aucun mécanisme d'échange entre les cégeps et l'université. En outre, j'avais oublié de mentionner que les étudiants ignorent la grammaire. Pour ce qui est de la culture générale, on ne s'en soucie guère.

P. H. - Je pense que nous sommes d'accord sur ces trois éléments de base : connaissance du français, connaissance de l'anglais et culture générale. Or, les enquêtes faites récemment sur l'enseignement des cégeps constatent des lacunes graves dans ces trois domaines...

F. W. - Une autre lacune grave est l'incapacité de penser de façon analytique, ce qui est désastreux pour le traducteur. Cette lacune se situe au niveau de la structure : beaucoup d'étudiants ont de la difficulté à rédiger une phrase structurée ; ils s'expriment par une série de phrases simples. On n'enseigne plus la connaissance formelle de la langue. Cela est également vrai dans le secteur anglophone et se rattache à des phénomènes comme ceux qu'a constatés McLuhan. Il se produit ainsi un décalage entre le monde de l'enseignement et le marché du travail, où on exige encore la qualité professionnelle ; et l'université est prise entre les deux. En ce qui concerne la «matière première », $j$ 'aimerais ajouter que le gouvernement fédéral a complètement faussé le processus des inscriptions en offrant des bourses aux finissants des cégeps. La situation a été partiellement corrigée depuis, mais pour beaucoup d'étudiants la motivation première est la bourse fédérale avec la garantie d'un travail d'été et d'un emploi.

G. G. - Il me semble que la situation est encore plus grave. Tout l'enseignement devrait être repensé très sérieusement. Le corps entier est atteint ; les remèdes «tampons » ne guérissent pas le mal chronique.

R.F. - Le phénomène constaté par M. Whyte n'est pas localisé mais universel. Nous vivons à l'ère de l'audiovisuel; les jeunes trouvent que les mots sont vides, qu'ils n'ont pas l'impact de l'image.

J. D. - On peut ajouter que l'étudiant québécois vit dans un milieu bilingue, ce qui concourt également à désarticuler sa pensée. Pour remédier à cette situation, il faudrait, à mon avis, enseigner les techniques de rédaction. 


\section{Le «produit fini »}

P. H. - Deux ou trois ans après son entrée à l'université, l'étudiant se présente sur le marché du travail. Comment se voit-il ? Comment est-il perçu par les autres, notamment par son employeur et ses collègues ?

J. D. - Vous avez raison de mettre «produit fini » entre guillemets, car il s'agit plutôt d'un produit semi-fini. Le travail de polissage doit se faire dans le milieu de travail, et ce n'est pas particulier à la traduction. L'employeur n'attend donc pas d'un finissant qu'il soit un as de la traduction. Il peut exiger par contre que le traducteur qu'il vient d'engager sache rédiger correctement et transposer convenablement un message, sans commettre les erreurs grossières d'un débutant.

A.M.D. - Ce qu'on exige, c'est un minimum raisonnable de connaissances au début, et ensuite un progrès constant. Il est également important que le traducteur se sente responsable de son texte ; trop de débutants s'en remettent au réviseur. Ils pensent : "Je produis mes 1500 mots, que le réviseur se débrouille », ou écrivent dans la marge « $\AA$ vérifier $» !$ La conscience professionnelle de chacun et les rapports entre traducteur et réviseur sont très importants.

P. H. - C'est pourquoi on a commencé à enseigner la révision dans le cadre des cours de traduction.

R.F. - En lisant «produit fini », je me suis demandé si c'était tel qu'il est ou tel qu'il devrait être; j'ai tenté de faire une synthèse des deux. On a l'impression que beaucoup de jeunes traducteurs nous arrivent comme poussés par un mauvais destin, et certains «tombent de haut». Ce qu'on attend, nous, c'est - pour citer Montaigne - une tête bien faite plutôt qu'une tête bien pleine, et cela me paraît encore plus important en traduction que dans les autres domaines. Il est normal qu'à son arrivée dans l'entreprise, l'étudiant se trouve dépaysé; il y trouve une hiérarchisation à laquelle il n'est pas habitué, il doit communiquer avec des supérieurs qui sont souvent anglophones, il s'aperçoit aussi qu'il appartient à une profession sans grand prestige, à un service souvent considéré comme un mal nécessaire ; enfin, il a de la difficulté à relier la traduction à la notion de profit, et donc à ne pas traduire pour la beauté de l'art.

J. D. - L'absence de prestige est une particularité importante de la profession. Dans certains milieux, on accole au traducteur l'étiquette peu flatteuse de glorified clerk.

R. F. - Et le Service de traduction est considéré comme un service auxiliaire, au même titre que la reprographie! Il y a aussi le gros problème du rapport qualité/quantité. À l'université, on a beaucoup insisté sur la qualité ; or, même si on retrouve ce souci de la qualité dans certains services de traduction, il reste que dans bien des cas c'est encore la quantité qui prime. Pour les relations entre traducteur et réviseur, j'ajouterai seulement que le traducteur doit avoir un sens poussé des relations humaines et une grande faculté d'adaptation.

A. M. D. - Oui, dans l'entreprise la traduction demande beaucoup de diplomatie. 
R. F. - Le traducteur doit savoir travailler de façon autonome, faire toutes les recherches nécessaires et aller au bout de ses moyens avant de déranger ses collègues. Je ne reviendrai pas sur la formation générale. Il doit avoir aussi un esprit de synthèse pour avoir une vue d'ensemble de son activité, connaitre la destination et l'utilisation du texte traduit pour ne pas accorder la même importance à une lettre destinée à une seule personne qu'à un contrat publié à des milliers d'exemplaires.

G. G. - La question des relations entre traducteur et réviseur peut aller très bin. Pour beaucoup d'étudiants stagiaires, le réviseur est l'ennemi, celui qui met des bâtons dans les roues, qui n'est jamais satisfait de la traduction produite. Le traclucteur en vient à douter de l'utilité de son travail, surtout lorsqu'il s'aperçoit que la plupart des textes qu'il traduit ne serviront à rien. On traduit pour la forme, pour le principe, et c'est décourageant.

P. H. - Je pense que vous venez de soulever un point très important, même s'il concerne surtout la Fonction publique. Je considère personnellement que la politique actuelle de bilinguisme est néfaste pour le traducteur et la traduction; dans certains cas, que je pourrais citer, c'est de l'aberration. Or, si en plus des problèmes de motivation qu'on a déjà évoqués, de toutes les difficultés que crée le milieu du travail, le traducteur constate l'inutilité de son travail, on aboutit vite à une situation impossible.

G. G. - Et il ne faut pas s'étonner de rencontrer chez les traducteurs beaucoup de gens bizarres, pour ne pas dire plus!

A. M. D. - Il y a aussi le fait que les traducteurs, à la différence des administrateurs, par exemple, n'ont pas de plan de carrière. On devient traducteur 1, $2,3,4$, puis réviseur et chef de section. C'est limité. Les employeurs encouragent peu ou pas l'initiative et le sens des responsabilités.

P. H. - C'est le cas dans la Fonction publique. J'aimerais demander à M. Frenette ce qu'il en est dans l'entreprise privée.

R. F. - Chez nous, la traduction mène à tout. J'ai dit que certains traducteurs avaient l'impression d'arriver dans l'entreprise poussés par un mauvais destin. Pour ceux-là, la formation reçue leur permet de bifurquer. Nous avons des traducteurs qui sont devenus relationnistes, rédacteurs au journal de l'entreprise, et même réalisateurs dans les studios de télévision en circuit fermé. Je pense qu'à l'avenir, les traducteurs pourront faire de l'adaptation et de la rédaction; nous nous sommes déjà engagés dans cette voie. Enfin, compte tenu de la situation actuelle au Québec, on va de plus en plus consulter les traducteurs sur des questions de langue, le service de traduction va devenir un centre de rayonnement du français dans l'entreprise. Et de plus en plus on fera de la rédaction parallèle au lieu d'une simple traduction.

P. H. - Nous nous éloignons un peu du sujet, mais je crois qu'il était important d'aborder ces différents aspects qui font partie intégrante de l'activité traduisante au Canada et dont il faudra tenir compte dans la formation des traducteurs. 


\section{Y a-t-il surproduction?}

Comme nous l'avons vu, le nombre des universités canadiennes dispensant l'enseignement de la traduction a plus que doublé en vingt ans. Les effectifs d'étudiants et de diplômés ont, évidemment, augmenté en proportion. En l'espace de cinq ans, (1970-1975), le nombre des étudiants à plein temps est passé de 200 à 1000 , et celui des diplômés, de 70 à plus de 300 . Si on ajoute les cours du soir, on arrive en 1975 à un total d'au moins 1750 étudiants.

Du côté de la demande, il y eu aussi unc progression rapide, notamment à la suite de l'adoption de la loi sur les langues officielles, et on peut penser que la loi 22 aura les mêmes effets au Québec. Citons un chiffre significatif : les effectifs du Bureau des traductions, qui étaient de 700 en 1973, devraient passer à 2000 en 1976-1977. Il semble néanmoins que l'offre reste inférieure à la demande, surtout si l'on considère qu'environ $80 \%$ des traducteurs engagés par le Bureau des traductions ne sont pas des diplômés. Y a-t-il donc un risque de surproduction?

G. G. - On peut le penser, si on en juge par le traitement des traducteurs à Ottawa. Après avoir connu une courbe ascendante, les salaires viennent de baisser, et je me demande s'il ne se passera pas la même chose qu'en droit.

F. W. - Il est vrai que les inscriptions en $1^{\text {re }}$ année ont augmenté considérablement, mais il faut aussi tenir compte du taux de désistement. Il est possible que le nombre des diplômés n'augmente pas en relation avec le nombre des inscriptions.

J. D. - En discutant avec les étudiants, j'ai constaté qu'un grand nombre d'entre eux ont l'intention de se diriger vers d'autres secteurs : journalisme, rédaction, relations publiques, etc. En outre, le taux de rotation du personnel est très élevé au Bureau des traductions. En ce moment, par exemple, beaucoup de traducteurs passent à l'École de langues de la Fonction publique. Les chiffres cités par M. Horguelin n'ont rien d'inquiétant, à mon avis, à condition que les universités n'abaissent pas leurs normes de qualité pour satisfaire une demande pressante.

F. W. - Je pense que les universités essaient de rationaliser l'enseignement et non d'en abaisser le niveau.

P. H. - Les chiffres bruts peuvent inquiéter, mais lorsqu'on les analyse, il semble en effet que la situation n'a rien d'alarmant. Je crois, à cet égard, qu'il serait utile d'avoir des statistiques sur le nombre des diplômés qui, après cinq ou dix ans, exercent encore la profession.

\section{Perfectionnement et recyclage}

P. H. - Parallèlement aux cours de formation que dispensent les universités, il existe des cours de perfectionnement qui s'adressent à des traducteurs en exercice. Ces cours se donnent le soir, dans le cadre de l'éducation permanente, et, plus récemment, sous l'égide des associations professionnelles. Que pensez-vous de cet enseignement?

J. D. - Les personnes qui entrent dans la profession sans avoir suivi de cours de traduction sentent très rapidement le besoin de se perfectionner; c'est à ces personnes que doivent s'adresser en premier lieu les cours de perfectionnement. Je 
vois aussi ces cours comme un moyen de se spécialiser; c'est ici que devrait se situer la spécialisation dont on parlait plus tôt.

F.W. - Je pense que les cours du soir peuvent assurer une formation très valable, car ils donnent le temps de faire une intégration des connaissances. Mais j'ai aussi lu l'article de l'Antenne qui les remet en cause.

P. H. - Le problème vient peut-être du fait que l'on s'adresse en même temps à trois publics à qui l'on offre le même enseignement : des jeunes qui viennent chercher une formation, des praticiens qui veulent se perfectionner et des personnes qui changent d'orientation.

J. D. - Il y a entre ces trois catégories des différences fondamentales, et je pense que les démarches entreprises par les sociétés professionnelles pour faire reconnaître l'exclusivité du titre ou de l'acte vont changer les perspectives.

P. H. - Oui. À ce sujet, le président de l'Office des professions du Québec a, dans une causerie récente, rappelé un point important du Code des professions. Il est notamment prévu que les corporations professionnelles devront offrir à leurs membres des cours ou des stages de formation continue, en collaboration avec le milieu de l'enseignement. Un comité d'inspection professionnelle pourra imposer des stages et même limiter le droit d'exercice pendant leur durée. Le perfectionnement ne sera donc plus laissé à l'initiative du traducteur, mais, dans certains cas, deviendra une obligation.

Une autre forme d'enseignement semble prendre de l'expansion depuis peu : la formation en vue du recyclage. Cette nouvelle orientation s'explique par la conjonction de deux faits : création de nouveaux débouchés en traduction et saturation de certains secteurs (sciences, enseignement...) C'est évidemment un changement radical, car au lieu de former des traducteurs qui ensuite se spécialisent, on donne une formation de traducteur à des personnes déjà spécialisées. Quels avantages et inconvénients voyez-vous à cette forme d'enseignement?

A. M. D. - Le gouvernement fédéral favorise cette formation en accordant des bourses; les résultats sont assez satisfaisants.

F. W. - C'est une réponse spécifique au problème de la spécialisation : on spécialise des traducteurs en fonction de besoins précis. Mais cette formule ne remplace pas la nécessité de former des traducteurs comme on le fait actuellement.

A. M. D. - Il y a quand même un risque. Il faut que l'étudiant spécialisé en biologie, par exemple, soit assuré d'un débouché à la fin de ses études ; sinon, il sera employé comme généraliste et ne fera peut-être pas un bon traducteur.

F.W. - Un autre danger, c'est que le diplômé d'une autre discipline a déjà acquis une façon de penser bien définie par sa spécialité. Je me demande si, même après sa formation de traducteur, il pourra adopter l'optique de la traduction qui lui sera nécessaire s'il doit abandonner sa spécialisation. C'est pourquoi je pense que ce genre de formation répond à une situation.

Orientation de l'enseignement

P. H. - Essayons maintenant de nous tourner vers l'avenir pour préciser qu'elle pourrait être ou devrait être l'orientation de l'enseignement de la traduction. 
Une première constatation s'impose, je crois, c'est l'absence de recherche, au Canada, dans le domaine des théories de la traduction, des méthodes d'enseignement, du matériel pédagogique. Comment remédier à cette lacune ? Avant de vous céder la parole, je crois utile de vous lire ce que M. Glaus, directeur de l'École de traducteurs et d'interprètes à l'Université d'Ottawa, écrivait dernièrement dans le bulletin Information:

Afin de garantir aux étudiants une formation appropriée, il est essentiel et urgent que les écoles consacrent une partie importante de leurs efforts à la recherche. Cette recherche doit porter à la fois sur les méthodes et les programmes d'enseignement et sur les méthodes et les outils de travail du traducteur et de l'interprète. L'école qui manquerait à la nécessité de faire de la recherche ne saurait maintenir sa place comme lieu d'enseignement valable en traduction ou en interprétation.

J. D. - Le nombre insuffisant de professeurs à plein temps est, à mon avis, une des principales raisons de l'inexistence de la recherche en traduction. D'autre part, la recherche théorique n'est valable que si elle trouve des applications pratiques qui fassent avancer la discipline. Or, jusqu'ici on s'est limité, au Canada, à explorer le domaine de la traduction automatique. Ces recherches jettent un nouvel éclairage sur certains problèmes de traduction, et c'est pourquoi il est bon que les conclusions de ces travaux soient communiquées aux étudiants. Mais il faut aussi explorer les avenues de la méthodologie et de la pédagogie de la traduction. Il faut que cette recherche soit concrète, solidaire des programmes d'enseignement de la traduction, qu'elle soit un approfondissement de la façon de faire passer l'expérience d'un traducteur à des étudiants qui doivent acquérir cette expérience. Par exemple, est-ce qu'un enseignement basé exclusivement sur la traduction de textes est la solution idéale ? Est-ce qu'un véritable manuel d'enseignement de la traduction est concevable? Quelle forme pourrait prendre un enseignement plus systématique? Il faudra répondre à toutes ces questions si l'on veut faire sortir l'enseignement de la traduction du stade artisanal.

F. W. - Je trouve cette préoccupation extrêmement intéressante, car à TroisRivières nous venons justement d'établir un projet de recherche qui s'oriente un peu dans cette direction. Notre idée de départ, c'est qu'on ne sait pas ce qui se passe à l'intérieur d'un programme de traduction. Chacun a son idée, mais on ne dispose d'aucune statistique. En plus, on arrive devant des représentants du Bureau fédéral qui nous disent que tout ce qu'on fait ne vaut pas grand-chose. C'est sans doute une exagération, mais il y a quand même quelque chose qui cloche. Et voilà que la traduction s'enseigne maintenant dans les cégeps, sans que soient précisés l'orientation et les objectifs de cet enseignement... Personnellement, je pense qu'on n'arrivera à rien tant qu'on n'aura pas suivi systématiquement le cheminement d'un étudiant en traduction. Dans le cas de la maîtrise, par exemple, on a la possibilité de contrôler les entrées, mais on n'a pas de critères pour le faire.

J. D. - Le moment est venu d'établir des critères d'évaluation des programmes et des étudiants.

A. M. D. - Il faut absolument qu'il y ait progression. À l'école où je suis allée, la plupart des étudiants avaient l'impression de perdre leur temps, de ressasser les mêmes choses. 
J. D. - L'établissement de critères d'évaluation précis sera, par contre, difficile à réaliser. Premièrement, la traduction couvre tout le champ des connaissances. Deuxièmement, il faut tenir compte du bagage de l'étudiant à son arrivée à l'université. Troisièmement, il faut considérer le talent de l'étudiant. Ce sont autant de facteurs difficilement quantifiables.

R. F. - Pour en arriver à une certaine stabilité des programmes, il serait peut-être utile de faire un sondage auprès des diplômés en leur demandant d'évaluer les cours qu'ils ont suivis, à la lumière de leur expérience pratique.

P. H. - On l'a fait il y a quelques années, et les professeurs ont trouvé très intéressant le rapport rédigé par ces diplômés. Mais je pense, comme M. Whyte, que les modifications de programmes doivent s'inscrire dans un ensemble cohérent, s'inspirer d'une "philosophie », sinon on retombe dans les solutions ad hoc.

F. W. - La raison pour laquelle ce système de changement perpétuel existe, c'est qu'on ne peut donner en trois ans la formation que tout le monde souhaite. Il faut faire un choix, et pour cela avoir des critères. Or, le seul qu'on ait, c'est celui de l'échec.

A. M. D. - Il ne faut pas oublier non plus, dans l'hypothèse où la recherche permettrait de découvrir des solutions nouvelles et de rationaliser les programmes, que l'administration universitaire a, elle aussi, des critères (rentabilité, concurrence entre universités) qui contredisent certains objectifs pédagogiques.

P. H. - Toujours dans une perspective d'avenir, pensez-vous que l'enseignement alterné soit une formule valable qui pourrait régler le problème du cloisonnement entre l'enseignement et la pratique?

J. D. - Cette formule peut être intéressante, à condition que les étudiants fassent un vrai stage et qu'on ne les «parque » pas dans une immense salle commune, comme cela se fait dans la Fonction publique. Il est essentiel que l'employeur sache ce qu'on attend de lui et qu'il accepte de collaborer à la formation de l'étudiant.

R. F. - C'est essentiel. Il faut que l'employeur ait les rèssources humaines et matérielles pour assurer à l'étudiant un stage formateur.

P. H. - Voyez-vous des difficultés d'ordre pratique pour l'entreprise ?

R.F. - Il faudrait d'abord qu'on soit averti suffisamment d'avance, pour inscrire le stage au budget. Il faudrait aussi que la période de stage soit fixée après accord entre les entreprises et l'université. Par exemple, février et mars, c'est pour beaucoup la période des rapports annuels et on est débordé. On aimerait aussi savoir quel est l'objectif précis que poursuit l'étudiant : faire des recherches, rédiger un mémoire, apprendre une terminologie. Enfin, le stage d'un mois est trop court, le stagiaire a à peine le temps de s'adapter.

A. M. D. - J'ai déjà eu à m'occuper de stagiaires à titre de réviseur. Or, nous n'avions reçu aucune directive, ni de l'employeur ni de l'université. Nombre de stagiaires, dans ces conditions, ne sont pas encadrés ; résultat : on les « occupe ». 
F. W. - Je regrette que les stages dont vous parlez n'existent qu'à l'Université de Montréal. Vous êtes privilégiés, de même qu'Ottawa. Même si le système d'enseignement alterné est intéressant, il présenterait des difficultés à Sudbury, à Moncton ou à Trois-Rivières. On risque alors de créer une élite à l'intérieur du corps étudiant : ceux qui ont eu la chance de faire un stage. Si donc on considère que le stage est nécessaire à la formation du traducteur, il faudra trouver des moyens pour l'offrir à tous les étudiants.

J. D. - À Ottawa, nous avons envisagé la possibilité que les professeurs s'occupent d'un groupe de stagiaires non boursiers à la Fonction publique pendant l'été. Cette formule serait ouverte à toutes les universités.

A. M. D. - Ce serait aussi formateur pour les professeurs, qui souvent sont coupés du milieu du travail. Mais je crains que, pour les stagiaires, on crée un milieu artificiel, ce qui serait contraire au but du stage.

F. W. - Nous, nous avons pensé à la formule inverse : faire venir un réviseur d'Ottawa qui dirigerait un stage à Trois-Rivières, pas nécessairement à l'université, dans des conditions qui seraient aussi proches que possible du milieu de travail. C'est un compromis, mais il me semble préférable à une situation où certains étudiants font un stage, et d'autres pas.

R.F. - L'université devrait aussi faire de la publicité autour des stages, montrer aux employeurs qu'ils ont un rôle à jouer, pour que le stage de traducteur devienne aussi normal que le stage de comptable ou l'internat.

P. H. - Dans un autre ordre d'idée, envisagez-vous possible que les écoles de traduction acceptent de spécialiser leur enseignement? Avec la multiplication des écoles et des options, on risque peut-être de disperser les énergies et les compétences, sans parler des investissements, au détriment de la qualité de l'enseignement.

A. M. D. - Surtout dans le cas des professeurs à plein temps. Pour les professeurs à temps partiel, un autre problème qui se pose est celui du manque de coordination. Là encore, l'administratif nuit au pédagogique.

G. G. - Depuis un certain temps, dès qu'on envisage une possibilité ou une solution, vous faites valoir qu'elle n'est pas réalisable pour des raisons administratives. Je me demande si l'enquête auprès des diplômés, dont vous parliez tout à l'heure, ne se heurterait pas au même obstacle. J'ai également retenu la nécessité d'une gradation dans l'enseignement, mais j'ajouterai que si on la prévoit sur le papier, il faut qu'on la retrouve au niveau des professeurs. Dans un même domaine, un cours de troisième année devrait être supérieur à celui de deuxième année. Si tel n'est pas le cas, le niveau baisse automatiquement et l'étudiant fait marche arrière.

A. M.D. - Je pense qu'à la défense des professeurs, il faut dire qu'ils sont considérés comme une quantité négligeable. Ils sont engagés à la dernière minute, on leur donne n'importe quel cours...

P. H. - Je vais vous demander maintenant d'apporter des conclusions à ce vaste tour d'horizon de la formation des traducteurs au Canada. 


\section{Conclusions}

F. W. - Je crois que le progrès dépend des sociétés professionnelles. Si elles arrivent à s'imposer, à faire respecter des normes, cela facilitera bien des choses : stages, prestige, motivation... Les problèmes de la formation universitaire ne seront pas d'office résolus, mais il sera plus facile de trouver des solutions.

A. M. D. - De leur côté, les sociétés professionnelles comptent sur les universités; elles ne pourront s'imposer que si les écoles de traduction sortent des éléments de valeur. On risque donc de se renvoyer la balle. Je crois néanmoins à la nécessité d'établir des rapports entre la profession et l'université.

P. H. - C'est d'ailleurs prévu par le Code des professions.

R. F. - Il me paraît indispensable qu'il y ait de plus en plus d'échanges entre la société professionnelle, les universités et les entreprises qui emploient des traducteurs. Il faudrait qu'elles se consultent plus souvent, que chacun mette de l'ordre dans sa maison et qu'ensemble on trouve des solutions.

G. G. - Et il ne faudrait pas oublier les étudiants, qui parfois ont l'impression que tout se décide sans eux. Or, c'est nous qui faisons les frais des erreurs d'aiguillage... Je souhaite que toutes les bonnes paroles que vous venez de prononcer ne restent pas de vains mots.

J. D. - En conclusion, je dirai que l'amélioration de la formation des traducteurs doit passer par une systématisation de l'enseignement. Il faut structurer davantage et établir une gradation plus nette des programmes, élaborer des critères précis pour évaluer les cours et les étudiants. L'enseignement actuel est bon, mais il demeure empirique. L'amélioration de la qualité de l'enseignement de la traduction doit en outre s'appuyer sur une recherche bien organisée. Celle-ci ne pourra être menée à bien sans un effort conjugué des universités et l'abandon d'un certain esprit de clocher. 